\title{
Das strategische Potenzial des Briefes: Friedrich Melchior Grimms Correspondance littéraire zwischen Privatbriefkultur und Pressewesen
}

Le potentiel stratégique de la lettre : la Correspondance littéraire de Friedrich Melchior Grimm entre civilité épistolaire et presse imprimée

The strategic potential of the letter: Friedrich Melchior Grimm's

Correspondance littéraire between epistolary culture and printed press

Jonas Hock

\section{OpenEdition}

Journals

Édition électronique

URL : http://journals.openedition.org/ceg/869

DOI : $10.4000 /$ ceg.869

ISSN : 2605-8359

Éditeur

Presses Universitaires de Provence

Édition imprimée

Date de publication : 30 mai 2016

Pagination : 71-82

ISBN : 979-10-320-0067-0

ISSN : 0751-4239

\section{Référence électronique}

Jonas Hock, « Das strategische Potenzial des Briefes: Friedrich Melchior Grimms Correspondance littéraire zwischen Privatbriefkultur und Pressewesen », Cahiers d'Études Germaniques [Online], 70 | 2016, Online erschienen am: 30 November 2017, abgerufen am 26 November 2020. URL : http:// journals.openedition.org/ceg/869; DOI : https://doi.org/10.4000/ceg.869 


\title{
Das strategische Potenzial des Briefes \\ Friedrich Melchior Grimms Correspondance littéraire zwischen Privatbriefkultur und Pressewesen
}

\author{
Jonas HOCK \\ Universität Regensburg
}

\section{Von Büchern, Käse und anderen Briefbeilagen - der literarische Korrespondent}

Das 18. Jahrhundert kannte eine besondere Figur des Briefeschreibers: den literarischen Korrespondenten. Dessen Aufgabe bestand darin, seinen fürstlichen Auftraggeber gegen teils recht fürstliche Bezahlung über literarische Neuheiten, die wichtigsten Theater- und Musikaufführungen, politische Entwicklungen, philosophische Diskussionen, Modetrends und selbstverständlich über den neusten Salonklatsch aus Paris zu informieren. Nicht selten ging der Auftrag soweit, die wichtigsten Bücher, Noten oder Abschriften schwer zugänglicher Texte, letztlich jede Art von „objet de Paris“1 gleich mitzuschicken. Häufıg betrafen die Bestellungen die neuste Mode: von Perücken über allerlei Accessoires bis hin zur vollständigen Ausstattung, durch welche die Empfänger, Adelige in ganz Europa an mehr oder weniger provinziellen Höfen, nicht nur ihren Geschmack, sondern auch ihre guten Kontakte nach Paris herausstellen konnten. Friedrich II. ging sogar soweit, sich von seinem literarischen Korrespondenten NicolasClaude Thieriot Käse schicken zu lassen, was Voltaire zu folgenden spöttischen Versen inspirierte:

\footnotetext{
Envoyer au roi des fromages,

Et les emballer prudemment

Dans certains modernes ouvrages. ${ }^{2}$
}

Einige Verse weiter ist im selben Gedicht von einem weiteren Charakteristikum der correspondances littéraires die Rede:

1. François Moureau, "Correspondants et correspondances allemandes de Paris à l'époque de Grimm " in Michel Delon, Jean Mondot (Hrsg.), L’Allemagne et la France des Lumières. Deutsche und französische Aufklärung. Mélanges offerts à Jochen Schlobach par ses élèves et amis, Paris, Honoré Champion, 2003, S. 167-183, hier $168 \mathrm{f}$.

2. „Dem König Käse schicken / Und diesen behutsam / In einige moderne Werke einpacken“; Voltaire, CEuvres. Vol. 54. Correspondance, t. 4, herausgegeben von Adrien-Jean-Quentin Beuchot, Paris, Lefèvre, 1831, S. 444. 
Thieriot me dit tristement :

Ce philosophe conquérant

Daignera-t-il incessamment

Me faire payer mes messages ? ${ }^{3}$

Der Korrespondent schickte seine Briefe in der Regel weder aus Freundschaft noch aus reinem aufklärerischem Idealismus - auch wenn beides nicht ausgeschlossen war. Eine literarische Korrespondenz war vor allem ein finanzielles Projekt, etwa Nebenverdienst für unterbezahlte Diplomaten oder notorisch finanzschwache Literaten, die so ihre guten Kontakte zu aufklärerischen Salonkreisen, ihre intime Kenntnis des intellektuellen und kulturellen Lebens in Frankreich oder auch ihre bloße Anwesenheit in Paris versilbern konnten.

Die ,Geburt" des literarischen Korrespondenten ging einher mit der Entstehung eines spezifischen Mediums, der correspondance littéraire. Diese ähnelte einer Zeitschrift, wurde aber handschriftlich verfasst, oft als Diplomatenpost verschickt, ohne jedoch primär außenpolitische Mitteilungen zu enthalten, und war häufig von einem einzelnen Absender an einen bestimmten Empfänger gerichtet, wobei es sich gerade nicht um einen privaten Briefwechsel handelte. Insofern war die literarische Korrespondenz, die correspondance littéraire, ein Medientypus, der als Randphänomen der europäischen Briefkultur in der zweiten Hälfte des 18. Jahrhunderts verstanden werden kann.

\section{Mehr als handschriftliche Zeitungen - Merkmale literarischer Korrespondenzen}

Von Jochen Schlobach, dem deutschen Pionier auf dem Feld der Erforschung literarischer Korrespondenzen des 18. Jahrhunderts, stammt der wohl erste Versuch, eine umfassende Bestimmung und Beschreibung der correspondance littéraire als Gattung vorzunehmen, als hybrides Genre, das zwischen Privatkorrespondenz und Zeitschriftenwesen anzusiedeln wäre. Aus seiner Untersuchung verschiedenster literarischer Korrespondenzen kondensiert er acht Merkmale, die den meisten seiner Quellen gemein sind. ${ }^{4}$ Mit gedruckten Periodika teilen die correspondances die Merkmale (1) Periodizität der Lieferung, (2) Bezahlung des ,Abonnements;, (3) häufig Festlegung eines Normumfangs, (4) standardisierter Aufbau, (5) unpersönlicher Charakter der Berichterstattung; dem Privatbrief ähneln sie v.a. durch die äußere Form, nämlich (6) Handschriftlichkeit und (7) individuelle Adressierung in Briefform; außerdem

3. ,Thieriot fragt mich trübselig: / Wird dieser eroberungslustige Philosoph [gemeint ist Friedrich II.] / die Güte haben, / meine Botschaften dauerhaft zu bezahlen?‘ Ibid., S. 445.

4. Die von ihm untersuchten und edierten correspondances sind versammelt im Band: Jochen Schlobach (Hrsg.), Correspondances littéraires inédites. Études et extraits. Suvies de Voltairiana, Paris/Genève, Champion-Slatkine, 1987; die acht aufgeführten Merkmale entnehme ich seinem einführenden Artikel im selben Band: "Les correspondances littéraires et le rayonnement européen de la France au XVIII e siècle ", S. 31-45, hier v.a. S. 39. 
sind (8) die Empfänger beinahe ausschließlich Adelige außerhalb Frankreichs, für die ,ihre ' literarische Korrespondenz der direkte Informationskanal aus Paris ist.

Die (1) Periodizität muss man sich als eine relative vorstellen: Die Sendungen waren datiert, was jedoch durchaus auch Rückdatierungen zur Wahrung eines Scheins von Regelmäßigkeit und zur Einhaltung der Abmachungen ermöglichte. Eine correspondance littéraire konnte allerdings auch individuelleren Rhythmen folgen, je nach zeitlichen Kapazitäten des Korrespondenten, evtl. seinen finanziellen Bedürfnisse oder abhängig von besonderen, für seine(n) Empfänger möglicherweise interessanten Ereignissen.

Die (2) Preise für literarische Korrespondenzen waren sehr unterschiedlich. Henri Duranton vermutet, dass der Preis für eine ,Standard'-Korrespondenz in etwa an dem für gedruckte Zeitschriften wie dem Mercure orientiert war. ${ }^{5}$ Der oben erwähnte Thieriot erhielt von Friedrich II. für seine Dienste jährlich immerhin 1200 livres; ${ }^{6}$ Grimm verlangte mithin bis zu 2000 livres im Jahr, was ca. zehn Arbeiterjahreslöhnen entsprach ${ }^{7}$ und ihm zu beträchtlichem Wohlstand verhalf, hatte jedoch keinen ,Festpreis‘, sondern machte die Summe von den finanziellen Möglichkeiten der Empfänger abhängig. ${ }^{8}$

Der (3) Umfang und die (4) Struktur einer correspondance littéraire-Lieferung konnten stark variieren: vom einzelnen Blatt bis hin zur zweistelligen Anzahl, von der Übermittlung dreier knapper Hinweise über die Kombination eines langen Artikels mit kleineren Notizen ${ }^{9}$ bis hin zur umfangreichen Sammlung von Texten verschiedenster Art und Provenienz wie im Falle von Grimms Correspondance littéraire, philosophique et critique.

Schlobach betont ebenfalls den (5) unpersönlichen Charakter der Schreiben. Nicht zuletzt aus pragmatischen Gründen wurde - vor allem wenn es sich um correspondances handelte, die in Abschrift an mehrere Empfänger gingen - auf die übliche Briefetikette, auf Anrede und Schlussformeln verzichtet, während das Datum durchgehend angeführt war. Ausnahmen bildeten beispielsweise Thieriot, der - ob in einem correspondance-Schreiben an Friedrich II. oder an den Fürst von Nassau-Usingen ${ }^{10}$ - an den üblichen Höflichkeitsfloskeln festhielt, oder eine im freundschaftlichen Ton gehaltene Sendung von Nicolas Maurice Chompré an François-Antoine de Boissy d'Anglas, den er als „mon bon ami“ anredet, um mit „Le meilleur des amis“ zu signieren ${ }^{11}$, was bei den anderen, meist von

5. Vgl. Henri Duranton, « Le genre de la correspondance littéraire et les moyens de communication intellectuelle dans l'Europe des Lumières ", in Schlobach, Correspondances littéraires inédites, S. 9-20, hier S. 9, Fn. 3.

6. Vgl. Schlobach, "Les correspondances littéraires », S. 37.

7. Vgl. Moureau, "Correspondants et correspondances allemandes ", S. 170.

8. Vgl. Ulla Kölving, "Introduction générale", in dies. (Hrsg.), Friedrich Melchior Grimm. Correspondance littéraire. Tome 1. 1753-1754, Ferney-Voltaire, Centre International d'Étude du XVIII ${ }^{\mathrm{e}}$ siècle, 2006, S. XXI-LXXII, hier XXXI.

9. Wie Schlobach die Standardstruktur bestimmt, vgl. "Les correspondances littéraires ", S. 39.

10. Vgl. den Abdruck zweier Schreiben in Schlobach, Correspondance littéraires inédites, S. 139-144.

11. Ibid., S. $229 \mathrm{f}$. 
Subalternen an deutlich höherrangige Adelige adressierten Korrespondenzen unmöglich gewesen wäre.

Die (6) Handschriftlichkeit der literarischen Korrespondenzen war kein Selbstzweck, sondern diente in vielen Fällen der Umgehung der Zensur aller Druckerzeugnisse. Sie konnte aber auch Einmaligkeit suggerieren und stellte eine Art symbolischen Mehrwert gegenüber Gedrucktem dar, selbst wenn die Texte oft von Kopisten geschrieben waren. ${ }^{12}$ Der Sicherheit vor Zensur und Verfolgung durch Staat und Kirche diente auch die (7) Zustellung der correspondances in Briefform und nicht selten als diplomatische Post. Das schützte die Sendungen zwar nicht endgültig vor dem Zugriff der cabinets noirs, bedeutete aber für die häufig als Diplomaten in Paris akkreditierten literarischen Korrespondenten auch keinen Mehraufwand.

Die Tatsache, dass literarische Korrespondenzen vor allem (8) Kanäle von der ,Hauptstadt des 18. Jahrhunderts` zu peripheren Höfen darstellten, soll hier nur kurz erwähnt werden, würde sie doch eine umfangreichere kulturgeschichtliche Kontextualisierung des damals europaweiten Interesses an französischen Kulturgütern, Ideen der frankophonen Aufklärer, dem absolutistischen Hof in Versailles und schlicht auch an den oben angesprochenen Konsumgütern verlangen, mit denen man sich noch am provinziellsten kleinfürstlichen Hof als geschmackvoll auszeichnen konnte - vorausgesetzt man hatte einen rührigen literarischen Korrespondenten in Paris. Die Besonderheit dieser historischen Konstellation macht auch die Historizität der correspondance littéraire als Gattung oder - da dieser Begriff eher literatur- als medienwissenschaftlich fundiert ist - als Medientypus in der oben beschriebenen Form aus. So beschrieb bereits Schlobach die correspondance littéraire konzis als „Produkt eines präzisen historischen Moments und einer europäischen Konstellation, in der aufgeklärte Prinzen und französischsprachige Schriftsteller, die den Ideen der Aufklärung mehr oder weniger verbunden waren, sich anzogen“. ${ }^{13}$

\section{„Ein Bulletin literarischen und weltgefälligen Inhaltes" - Grimms Correspondance littéraire}

Die Correspondance littéraire, philosophique et critique ${ }^{14}$ des nach Paris ausgewanderten Regensburger Pastorensohns Friedrich Melchior Grimm gilt

12. Vgl. Duranton, "Le genre de la correspondance littéraire ", S. 13.

13. " [P]roduit d'un moment historique précis et d'une constellation européenne, où princes éclairés et écrivains de langue française plus ou moins rattachés aux idées des Lumières s'attiraient "; Schlobach, "Les correspondances littéraires ", S. 45.

14. Die bis heute einzige deutsche Teilübersetzung der Correspondance littéraire erschien 1977 in der DDR und gleichzeitig als Lizenzausgabe beim Münchner Hanser-Verlag in der BRD: Melchior Grimm, Paris zündet die Lichter an. Literarische Korrespondenz, aus dem Französischen von Herbert Kühn, herausgegeben von Kurt Schnelle, Leipzig, Dieterich'sche Verlagsbuchhandlung, 1977. Derzeit entsteht am Centre international d'études du XVIII 'e siècle in Ferney-Voltaire unter der Leitung von Ulla Kölving die erste historisch-kritische Gesamtausgabe der Correspondance 
unangefochten als bedeutendste literarische Korrespondenz. Goethe, der Grimms Correspondance sehr schätzte und dem „durch die Gunst hoher Gönner, eine regelmäßige Mitteilung dieser Blätter beschieden“ ${ }^{15}$ war, beschrieb sie wie folgt:

\begin{abstract}
Als vor vierzig Jahren Herr von Grimm sich in der damals ausgezeichneten geist- und talentvollen Pariser Gesellschaft einen ehrenvollen Zutritt gewonnen und für ein Mitglied eines so außerordentlichen Vereins wirklich anerkannt wurde, beschloß er ein Tagesblatt, ein Bulletin literarischen und weltgefälligen Inhaltes, schriftlich zu versenden an fürstliche und reiche Personen in Deutschland, um sie, gegen bedeutende Vergeltung, von dem eigensten Leben der Pariser Zirkel zu unterhalten, nach deren Zuständen man äußerst neugierig war, weil man Paris als den Mittelpunkt der gebildeten Welt wirklich ansehen konnte. ${ }^{16}$
\end{abstract}

1753 hatte Grimm wohl nicht, wie oft angenommen, eine correspondance littéraire von Guillaume ,Abbé Raynal übernommen, wohl aber nach und nach einige der Abonnenten von dessen Nouvelles littéraires. ${ }^{17}$ Mehr als zwanzig Jahre später übergab er sein Projekt dann an seinen langjährigen Sekretär Jakob Heinrich Meister, der es, weniger regelmäßig und mit Unterbrechungen, aber unter gänzlich anderen Vorzeichen, bis Ende 1813 fortführte. Für die mehr als 20 Jahre unter Grimms Leitung stellt die Correspondance eine weitgehend vollständige geistes- und kulturgeschichtliche Chronik der französischen Aufklärung dar. Gleichzeitig kann sie nicht als typische Vertreterin für den Medientypus ,literarische Korrespondenz، betrachtet werden. Obgleich Grimm manch andere literarische Korrespondenten inspirierte - die ihm sein prestigereiches Projekt durchaus neiden konnten -, wie Moureau beispielhaft an Gottlieb Schütze und Baron von Boden zeigt ${ }^{18}$, unterschied sich seine correspondance in verschiedenen Punkten von anderen.

Auch Grimm besorgte für seine Abonnenten Haarbänder, Uhren, Möbel ${ }^{19}$ und die Correspondance littéraire, philosophique et critique wurde (1) regelmäßig versandt. Der (2) hohe Preis für ein Abonnement sowie der (3) große Umfang der Lieferungen wurden bereits erwähnt; hier soll vor allem der (4) Aufbau einer Lieferung beispielhaft dargestellt und die (5) Entindividualisierung seiner Schreiben, d.h. der bewusste, strategische Verzicht auf Privatbriefnormen angesprochen werden; auch (6) Handschriftlichkeit, (7) Briefform und (8) Adressierung an Adelige waren konstitutiv.

littéraire zumindest für die Grimm'sche Periode, von der bisher neun Bände erschienen sind. Zur bewegten Editionsgeschichte des Periodikums - die erste Druckausgabe stammt von 1813 vgl. die bereits zitierte "Introduction générale " von Kölving, S. XXIX-L.

15. Johann Wolfgang Goethe, „Urteilsworte französischer Kritiker“, in ders., Sämtliche Werke in 18 Bänden. Band 14. Schriften zur Literatur, Zürich, Artemis, 1949, S. 778-785, hier 784. Durch seinen Zugang zur Correspondance littéraire konnte Goethe Werke wie Diderots Jacques le Fataliste oder La Religieuse lange bevor sie gedruckt wurden zur Kenntnis nehmen.

16. Ibid.

17. Vgl. Kölving, "Introduction générale », S. XXIX f.

18. Vgl. Moureau, "Correspondants et correspondances allemandes ", S. 171-183 sowie das auf diesem Artikel basierende Kapitel in François Moureau, La plume et le plomb. Espaces de l'imprimé et du manuscrit au siècle des Lumières, Paris, PUPS, 2006, S. 427-443.

19. Vgl. Schlobach, "Les correspondances littéraires », S. 34. 
Weitere zentrale Aspekte waren einerseits die kollektive Produktion der Correspondance sowie deren Erfolg bzw. Verbreitungs- und Wirkungsgrad, andererseits Grimms persönliches Engagement - während für viele andere eine literarische Korrespondenz eher Nebenprojekt oder finanzielle Übergangslösung war - sowie sein aufklärerischer Anspruch, der aus seinen Blättern ein Medium des Kampfes der philosophes werden ließ. ${ }^{20}$

Grimm erreichte mit seinen Sendungen zeitweise mehr als zwölf Abonnenten gleichzeitig, darunter u.a. Katharina II., den schwedischen König Gustav III., den polnischen König Stanislaw Poniatowski und für eine gewisse Zeit sogar Friedrich II. ${ }^{21}$ Dass es bei einer so umfangreichen correspondance der Arbeit von Kopisten bedurfte und weitgehend auf Briefform und -förmlichkeit verzichtet wurde, ist nachvollziehbar. Abrosimov zeigt jedoch auf, dass diese Form bei Grimm nicht nur pragmatisch motiviert war, sondern Teil seiner umfassenderen Kommunikationsstrategie, die darauf ausgerichtet war, einen geheimen, ,esoterischen“ und damit geschützten Kommunikationsraum für philosophes und princes éclairés zu schaffen, in dem radikalphilosophische Ideen diskutiert und gleichzeitig einflussreichen Herrschern gleichsam injiziert werden konnten. Grimms „programmatischer Bruch mit dem Briefzeremoniell“ zeigt sich sogar in der Blattgröße des verwendeten Papiers, für das er das mittlere Format $(230 \times 185 \mathrm{~mm})$ anstatt der für den Briefverkehr mit Fürsten vorgeschriebenen Größe $(320 \times 210 \mathrm{~mm})$ wählte, sowie in der „radikalen Abweichung vom geforderten Muster der ,belle écriture““, die „als eine Art Unabhängigkeitserklärung Grimms gedeutet werden“" ${ }^{22}$ könne - also im Sinne der Verweigerung formalästhetischer Unterwerfungsgesten.

Die Sicherung der Autonomie der Correspondance littéraire war auch Ziel ihrer einseitigen Gerichtetheit. Abrosimov weist nach, dass Grimm bewusst auf das Prinzip der Wechselseitigkeit verzichtete, das eigentlich unumstößliche Grundregel jeder Korrespondenz im Sinne eines Briefwechsels ist. Diesen lagerte Grimm jedoch in seine Privatkorrespondenz aus, die der individuellen Beziehungspflege mit seinen Abonnenten diente und allen Regeln des Briefverkehrs folgte. ${ }^{23}$ Was die Correspondance anging, so waren die Fürstinnen und Fürsten auf die Rolle passiver Empfänger festgelegt, deren Versuche der Einflussnahme oder gar der Mitarbeit Grimm erfolgreich mit Verweis auf das höhere kollektive Interesse oder das anderer Empfänger neutralisieren konnte.

20. Diese vier Punkte werden vortrefflich von Kirill Abrosimov in seiner kürzlich erschienenen Dissertation analysiert, einer medien- und kommunikationsgeschichtlichen Untersuchung der Grimm'schen Correspondance, auf die sich die folgende Skizze in großen Teilen stützt. Vgl. Kirill Abrosimov, Aufklärung jenseits der Öffentlichkeit. Friedrich Melchior Grimms ,Correspondance littéraire‘ (1753-1773) zwischen der, république des lettres‘ und europäischen Fürstenhöfen, Ostfildern, Thorbecke, 2014.

21. Vgl. Kölving, "Introduction générale ", S. XXXII ff.

22. Abrosimov, Aufklärung jenseits der Öffentlichkeit, S. 110.

23. Große Teile von Grimms Briefwechseln jenseits der Correspondance liegen mittlerweile in zwei Bänden vor. Vgl. Jochen Schlobach (Hrsg), Correspondance inédite de Frédéric Melchior Grimm, München, Wilhelm Fink, 1972 sowie Jochen Schlobach, Véronique Otto (Hrsg.), Correspondance privée de Frédéric-Melchior Grimm. 1723-1807, Genève, Slatkine, 2009. 
Auch wurde durch die fehlende persönliche Ansprache auf textlicher Ebene ein anonymes Empfängerkollektiv konstruiert. ${ }^{24}$

Dass diese Entindividualisierung vor allem strategischer Natur war, ließe sich auch daran ablesen, dass Grimm der Briefästhetik seiner Zeit durchaus nicht ablehnend gegenüber stand. Bernard Bray kommt bei seiner Untersuchung der Geschmacksurteile der Correspondance-Redakteure in ihren Kommentaren zu zeitgenössischen Briefromanen, Sammlungen authentischer Briefe und offenen Briefen (die in den Text der Correspondance selbst aufgenommenen Briefe beachtet er nicht) zu dem Ergebnis, „der Anspruch, [den Grimm] formuliert, ist ein dreifacher. Er betrifft zunächst die Authentizität des Textes [...], sodann die menschliche Bedeutung des Inhalts, schließlich die natürliche Schlichtheit des Stils“. ${ }^{25}$ Diese Orientierung an Authentizität und Natürlichkeit erinnert gleich auf den ersten Blick an Gellerts Ausrichtung des Briefes am mündlichen Gespräch; ${ }^{26}$ die textuelle Konstruktion der Correspondance littéraire-Lieferungen trug diesem Ideal allerdings keinerlei Rechnung.

Bray formuliert bereits recht emphatisch das grundlegende strategische Potenzial des Briefes, wie es von Grimm zum Einsatz gebracht wurde:

Letztlich spielt es keine Rolle, ob der Brief anonym oder unterzeichnet ist. Durch seine Form strebt er danach, an die Stelle der durch die Tradition erstarrten Ordnung anerkannter Werte die Ordnung einer Debatte zu setzen, die offen für alle ist und sich beständig fortentwickelt. ${ }^{27}$

Allerdings war die Correspondance littéraire eben nicht „ouvert à tous“ und funktionierte auch nicht gemäß einer gesprächsähnlichen Dialogizität im Sinne eines Austauschs zwischen Sender und Empfänger. Das war nicht nur Zensur und Verfolgungsdruck durch Staat und Kirche zuzuschreiben. Grimms Bestimmung der Correspondance als ,Sprachrohr der französischen philosophes - nicht für die Massen, sondern für den exklusiven Kreis bestimmter politischer Entscheidungsträger in ganz Europa, die für aufklärerisches Gedankengut bereits empfänglich waren - schloss den Dialog als Rollentausch von Schreibenden und Lesenden im Medium selbst aus. So konnte es kaum unmittelbare ,Leserbriefe der Adeligen geben, wohl aber eine Vielstimmigkeit, welche die Etablierung einer kohärenten Ideologie auf den Seiten der Correspondance verhinderte und ihren kollektiven Charakter auf der Produzentenseite widerspiegelte. Nicht nur verfasste Grimm seine Lieferungen teils gemeinsam mit Madame d'Épinay und Diderot, die ihn bei Abwesenheit sogar vertraten und die Redaktion

24. Vgl. Abrosimov, Aufklärung jenseits der Öffentlichkeit, S. 103-112.

25. « [L'] exigence formulée [par Grimm] est triple. Elle concerne d'abord l'authenticité textuelle [...], puis l'intérêt humain du contenu, enfın la simplicité naturelle du style " ; Bernard Bray, "La CL témoin du goût pour la forme épistolaire ", in ders., Jochen Schlobach, Jean Varloot (Hrsg.), La Correspondance littéraire de Grimm et de Meister (1754-1813), Paris, Klincksieck, 1976, S. 213-220, hier S. 215 f.

26. Es wäre durchaus lohnend, Grimm als Privatbriefschreiber im breiteren Kontext deutscher und französischer Briefkulturen und -traditionen zu untersuchen.

27. «Peu importe, finalement, que la lettre soit anonyme ou signée. Elle prétend en réalité par sa forme substituer à l'ordre des valeurs reconnues, figées par la tradition, l'ordre d'un débat ouvert à tous et en constant progrès. " Bray, "La CL témoin du goût pour la forme épistolaire ", S. 220. 
gänzlich übernahmen, sondern es wurden auch Texte verschiedenster Dritter - ob vom Ruf eines Voltaire oder unbekannter kleiner abbés - in den Text der Sendungen aufgenommen. Diese Briefe, Gedichte, Pamphlete oder Berichte unterschiedlichster Provenienz konnten einzelne Correspondance-Lieferungen $\mathrm{zu}$ einem wahren Flickenteppich machen, einem Gemenge an Meinungen, Urteilen und Wahrheiten, die nicht mit Grimms eigener Position übereinstimmen mussten, wodurch in einem einzigen Schriftstück einer Vielfalt an Verfassern und damit Perspektiven Raum gegeben wurde.

\section{Ein "Brief", viele Verfasser - die Vielstimmigkeit der Correspondance littéraire}

Als Beispiel für die Polyperspektivität von Grimms Correspondance soll im Folgenden die Lieferung vom 15. September 1761 dargestellt werden, die u.a. den Beginn von Diderots Salon de 1761 umfasst. Bereits Grimms einleitende Worte sind bemerkenswert:

L'Académie royale de peinture et de sculpture expose, comme vous savez, tous les deux ans les ouvrages de ses maîtres dans un salon du Louvre. [...] Il ne s'agit pas ici de vous répéter de fades détails dont tous nos journaux regorgeront pendant quelques mois ; mais de vous donner une idée exacte du travail de nos artistes; et pour m'acquitter de ce devoir, je cède la plume à un homme qui pouvant dire comme le Corrège : Ed anch'io son pittore, sait aussi mieux que personne mettre un prix équitable aux productions de ses confrères. M. Diderot qui est, à mon gré, le plus fort coloriste que nous ayons parmi les gens de lettres, m'a adressé sur le Salon les observations suivantes ; car vous savez qu'il faut toujours qu'il parle à quelqu'un. [...] C'est donc M. Diderot qui va parler. ${ }^{28}$

Auf die thematische Hinführung - ausgelassen wurden im Zitat Grimms Bemerkungen zu Dauer und Erfolg der Ausstellung - folgt eine Abgrenzung vom Journalismus der Printzeitungen, die nur die immer gleichen „faden Details“ wiederholen. Dagegen wird Diderot als Experte eingeführt, dessen Beobachtungen hier wiedergegeben werden; der Übergang zu Diderots Text wird überdeutlich markiert und dieser schließt sich unmittelbar an:

Voici, mon ami, les idées qui m'ont passé par la tête à la vue des tableaux qu'on a exposés cette année au Salon. Je les jette sur le papier sans me soucier ni de les trier, ni de les écrire. Il y en aura de vraies, il y en aura de fausses. Tantôt vous me trouverez trop sévère ; tantôt trop indulgent. Je condamnerai peut-être, où vous approuveriez. Je ferai grâce où vous condamneriez. Vous exigerez encore où je serai content. Peu m'importe. La seule chose que j'aie à cœur, c'est de vous épargner quelques instants que vous emploierez mieux, dussiez-vous les passer au milieu de vos canards et de vos dindons.

Louis Michel Vanloo

N. B. On pourra remarquer la grandeur des tableaux dans le petit livret.

28. Zit. nach Ulla Kölving (Hrsg.), Friedrich Melchior Grimm. Correspondance littéraire. Tome 8, 1761, Ferney-Voltaire, Centre International d'Étude du XVIII ${ }^{\mathrm{e}}$ siècle, 2013, S. 291-315, hier S. $291 \mathrm{f}$. 
Le premier tableau qui m'ait arrêté est le Portrait du roi. Il est beau, bien peint, et on le dit très ressemblant. ${ }^{29}$

Während Grimms Anrede („Sie wissen, dass ...“) sich kollektiv an seine Leserinnen und Leser zu wenden scheint, ist Diderots Text eindeutig an Grimm adressiert („mein Freund“), wodurch ersteren Empfängern hier der Status von privilegierten Mitlesenden zukommt, die dem - vermeintlich intimen und authentischen Austausch zweier Freunde beiwohnen können. Dabei enthält die zitierte Passage bereits drei redaktionelle Eingriffe Grimms, die Diderots Text den Gegebenheiten der Correspondance anpassen: Wie die äußerst hilfreichen Annotationen der Kölving-Ausgabe vermerken, ist der Rubrik- bzw. Kapiteltitel, d.h. der Name des Malers Vanloo hier wie bei den sechs folgenden Abschnitten vom Redakteur eingefügt. Auch das Notabene stammt von Grimm, der wohl allen Sendungen ein Ausstellungsheft beilegte. Ein Vergleich mit Diderots ursprünglichem Manuskript zeigt zudem, dass die Puter („dindons“) ursprünglich Entenküken („canetons“) waren und die Enten („canards“) von Grimm an die Stelle von „Dom Antonio“ gesetzt wurden; mit diesem Namen hatte Diderot ein Schwein auf dem Gehöft seines Verlegers Le Breton bedacht, was Grimms Leserschaft kaum hätte wissen können, weshalb der aufmerksame Redakteur ihr diese Chiffre ersparte. ${ }^{30}$

Nach einem knappen Absatz zu Vanloo folgen Kritiken zu den ausgestellten Werken von sechs weiteren Malern. Der letzte davon ist Noël Hallé, von dem Diderot gleich im ersten Satz feststellt: „Es gibt, meines Erachtens, nicht ein Stück von Herrn Hallé, das etwas wert ist."31 Abgeschlossen wird dieser erste Teil von Diderots Salon de 1761 mit der wohl wieder von Grimm eingefügten Bemerkung: „Die Fortsetzung folgt mit der nächsten ordentlichen Lieferung“32, wobei er das Urteil seines Freundes nicht unkommentiert lässt, sondern durch eine eigene Kritik eine alternative Meinung anbietet:

\footnotetext{
J'ajouterai, cependant, un mot sur ce dernier tableau que M. Diderot me paraît juger avec trop de sévérité. Cela vient, à ce que je crois, de ce qu'il a dans la tête, les sublimes compositions de Raphaël, de le Sueur et d'autres grands hommes dans des tableaux de prédication. M. Hallé n'est assurément pas un homme sublime, son tableau n'a nulle élévation, nul génie ; mais il a de la vérité [...]. ${ }^{33}$
}

Auf Grimms eigenen kunstkritischen Beitrag folgen dann diverse kürzere Notizen, die zum Großteil Neuerscheinungen vorstellen und mit scharfen, wertenden Kommentaren versehen. So wird das Buch eines anti-philosophe abqualifıziert, der, mit Bezug auf den oben von Diderot kritisierten Maler Hallé als „[e]in Militärangehöriger“ eingeführt wird, „der es verdient hätte, von Herrn Hallé gemalt zu werden“; und weiter heißt es: „Wenn dieser Armeeangehörige die Feinde des Königs nicht besser bekriegt als die Philosophen, so ist zu

29. Ibid., S. 292.

30. Ibid., Fn. 6 und 7.

31. " Il n’y a pas[,] à mon gré, un morceau de M. le professeur Hallé qui vaille. " Ibid., S. 306, Komma von Hrsg. eingefügt.

32. "La suite pour l'ordinaire prochain. " Ibid., S. 209.

33. Ibid. 
befürchten, dass er niemals Maréchal de France wird.“" ${ }^{34}$ Es folgen weitere Buchbesprechungen, sogar von einem Werk, das bereits über die Correspondance Verbreitung gefunden hatte: „Vor kurzem ist die Erzählung la Reine de Golconde gedruckt worden, die Sie vor einigen Monaten als Beilage zu diesen Blättern haben lesen können." ${ }^{35}$ Tatsächlich war diese Erzählung von Stanislas de Boufflers Teil der Lieferung vom 15. Juli desselben Jahres gewesen.

Auch der Brief und das darin enthaltene Gedicht eines jungen Benediktiners werden weitgehend kommentarlos, aber gekürzt wiedergegeben; wie die Herausgeberanmerkung anzeigt, war er an den Beichtvater der Mutter von Madame d'Épinay gerichtet, über den er wohl auch in Grimms Hände gelangte:

Lettre de don Le Fèvre, jeune bénédictin, à M. Martin curé de Deuil à trois lieus de Paris. De l'abbaye de St Thiery près Rheims. Ce 21 août 1761 .

Du haut d'un couteau champenois

Où mes errantes destinées,

Sans avoir consulté mon choix,

M'ont fixé pour quelques années,

$[\ldots]$

Permettez, monsieur, que je me remette au ton uniforme de la prose, et excusez, s'il vous plaît [,] dans nous autres provinciaux, le peu de talent que nous avons pour parler le langage des dieux. Je finirai donc par vous demander tout uniment, comment vous vous portez, et si vous pensez quelquefois à nous, en vous en informant par le père Charlemagne [etc.]. ${ }^{36}$

Abgeschlossen wird die Sendung vom 15. September 1761 mit einer Meldung aus der ,mondänen“ Welt des Theaters. Auch diese Nachricht über den Tod der jungen Schauspielerin Jacqueline Camouche wird von einem persönlichen Geschmacksurteil Grimms begleitet:

Kürzlich ist Fräulein Camouche gestorben, eine junge Schauspielerin von ungefähr zwanzig Jahren. [...] Sie machte eine schöne Figur auf der Bühne; aber ihr Talent war, meiner Ansicht nach, dürftig. ${ }^{37}$

Die folgende Lieferung vom 1. Oktober knüpft bei Diderots Kunstkritik an und bringt die Fortsetzung des Salon-Textes.

Diese Skizze einer konkreten Ausgabe der Correspondance littéraire sollte einerseits einen Eindruck von der thematischen Bandbreite der Meldungen vermitteln, andererseits ansatzweise die vielstimmige Textstruktur widerspiegeln, die in einer Sendung verschiedene Perspektiven widerspiegelte - einer moderierten Diskussion verschiedener Gesprächsteilnehmer nicht unähnlich. War Grimms literarische Korrespondenz letztlich also nichts anderes als ein

34. "Un militaire qui aurait mérité d'être peint par M. Hallé [...]. " " Si ce militaire ne fait pas mieux la guerre aux ennemis du roi qu'aux philosophes, il est à craindre qu'il ne soit jamais maréchal de France. "Ibid., S. 310.

35. "On a imprimé depuis peu le conte de la Reine de Golconde que vous avez lu, il y a quelques mois, à la suite de ces feuilles. "Ibid., S. 311.

36. Ibid., S. $313 \mathrm{f}$.

37. "Mademoiselle Camouche, jeune actrice de vingt ans environ, vient de mourir. [...] Sa figure était belle au théâtre ; mais ses talents, à mon gré, médiocres. "Ibid., S. 314. 
Salongespräch in Textform? Die Rede vom Brief als schriftlichem Gespräch war im 18. Jahrhundert üblich. ${ }^{38}$ Entsprechend diskutiert Abrosimov eine mögliche Konzeptualisierung der Correspondance als Salongespräch, worauf sogar einzelne Bemerkungen Grimms hinweisen. ${ }^{39}$ Doch gibt es auch anderslautende Äußerungen Grimms, da die Unterwerfung der Salondiskussion unter soziale Normen (wie Konfliktvermeidung) die Kritik entschärfe und zu Oberflächlichkeit zwinge - was er für sein Periodikum gerade vermeiden wollte. So war am Ende doch die Schriftlichkeit konstitutiv für den Anspruch, den Grimm an sein Projekt hatte - den kritischen und gelehrten Austausch, dessen Komplexität und Tiefe erst die Schriftlichkeit garantieren konnte.

\section{Vom Ende der literarischen Korrespondenzen}

Es sollte deutlich geworden sein, dass Grimms Correspondance littéraire bestimmte Aspekte der Briefform nutzte, um einen eigenen Kommunikationsraum zwischen der Exklusivität privaten Briefaustauschs und der Öffentlichkeit der gedruckten Presse zu schaffen. Die Historizität dieses ,Raumes“ bzw. dieser Kommunikationsstrategie darf nicht unterschätzt werden. Abrosimov deutet Grimms Projekt als „Antwort auf die tiefgreifende Krise der Gelehrtenrepublik in der zweiten Hälfte des 18. Jahrhunderts“ ${ }^{40}$, da die (ursprünglich humanistischen) Kommunikations- und Wissensproduktions-Ideale der République des Lettres von der Realität sozioökonomischer, v.a. technischer Umwälzungen überholt worden seien. Von seinem Ende her betrachtet wäre es hingegen gerade der weitergehende Wandel der politischen, medialen und (sozio-)ökonomischen Rahmenbedingungen, der den Zeitabschnitt der correspondances littéraires zum ausgehenden 18. Jahrhunderts hin nicht zuletzt mit der Französischen Revolution enden ließ. Der oben bereits zitierte „präzise historische Moment“, d.h. die „europäische Konstellation, in der aufgeklärte Prinzen und französischsprachige Schriftsteller, die den Ideen der Aufklärung mehr oder weniger verbunden waren, sich anzogen“" ${ }^{41}$, von der Schlobach spricht, war auf allen Ebenen Veränderungen unterworfen. Angedeutet sei hier nur die weitere Ausdifferenzierung von Briefkultur und Zeitschriftenwesen; die verstärkte Trennung von Privatsphäre und Öffentlichkeit in einer sich weiter auflösenden Ständegesellschaft; die allmähliche Auflösung des parti philosophique als gut vernetzter intellektueller Kerngruppe der französischen Aufklärung, der in ganz Europa mehr oder weniger aufgeklärte Fürsten gegenüberstanden; die geringer werdende einseitige Orientierung Europas an Paris. Duranton betont vor allem die Ausdifferenzierung der Wissensorganisation, da durch Spezialisierung die Einheit dessen, was einst den honnête homme, dann den philosophe oder den

38. Vgl. bspw. Robert Vellusig, Schriftliche Gespräche. Briefkultur im 18. Jahrhundert, Wien, Böhlau, 2000.

39. Vgl. Abrosimov, Aufklärung jenseits der Öffentlichkeit, S. 179-190, v.a. S. 181 f. und 187-190.

40. Ibid., S. 127.

41. Schlobach, "Les correspondances littéraires ", S. 45. 
prince éclairé interessieren konnte - also so gut wie alles, was Politik, Philosophie, Kulturproduktion im weitesten Sinne und auch Naturwissenschaftliches betraf -, nicht mehr gegeben gewesen sei; „Der Humanismus ist Enzyklopädie geworden, und diese Spezialisierung “. ${ }^{42}$

Der Bezug von correspondances littéraires wurde aber wohl ebenso wenig unmittelbar von Abonnements spezialisierter Zeitschriften abgelöst, wie die literarische Korrespondenz in gedruckter Form als Untergenre im professionellen Journalismus aufgegangen wäre. Dass Zeitschriftenartikel in Briefform erschienen, war bereits in der ersten Hälfte des 18. Jahrhunderts durchaus üblich und so ähnelten manche Texte in Periodika wie Le Pour et Contre des abbé Prévost oder dem Journal des Savants der Form nach einem Privatbrief, während sie inhaltlich-strukturell und vor allem stilistisch (journalistisch-trocken, ohne sentimentale Spuren) der literarischen Korrespondenz sehr nahe kamen. „[D] as Periodikum behält einen halbprivaten Charakter“43, wie Duranton vor allem an von Élie Fréron in den 1740ern und 50ern gegründeten Zeitschriften nachweist; dessen antiaufklärerische Journale Lettres de madame la Comtesse de ${ }^{* * *}$, Lettres sur quelques Écrits de ce temps oder L'Année littéraire trugen den Brief teils bereits im Titel und enthielten entsprechend in Briefform gehaltene Artikel. Nicht die formalen bzw. texttypologischen Bedingungen der gedruckten Presse waren es also gewesen, welche die literarische Korrespondenz in den Briefumschlag gezwungen hatten. Letztlich war es die kommunikations- und mediengeschichtliche ,Großwetterlage', welche die correspondance littéraire als kommunikationsstrategische Möglichkeit und Notwendigkeit innerhalb der Briefkultur des 18. Jahrhunderts für einen bestimmten Zeitraum hatte florieren und nach einer nachhaltigen Verschiebung auch wieder verblühen lassen.

42. " [L]'humanisme est devenu encyclopédie et celle-ci spécialisation "; Duranton, "Le genre de la correspondance littéraire ", S. 20.

43. "[L]e périodique garde un caractère semi-privé " ; ibid., S. 13 ; für die weiteren Ausführungen vgl. ibid. S. $10 \mathrm{ff}$. 